\title{
INVERSE LOGARITHM DECAY OF LONG-TERM DYNAMIC APERTURE IN HADRON COLLIDERS*
}

\author{
M. Giovannozzi ${ }^{\dagger}$, INFN, Sezione di Bologna, Italy \\ W. Scandale, CERN, Geneva CH 1211 \\ E. Todesco, Dipartimento di Fisica Università di Bologna, Italy \\ ripple effect [10, 11].
}

Abstract

The long-term behaviour of the non-linear single-particle dynamics is considered for the 4D Hènon map. The dynamic aperture is defined as the average of the particle loss boundary over several ratios of emittances. Using this definition, survival plots turn out to decay with the inverse of the logarithm of the number of turns. We also compare the extrapolation of the dynamic aperture with the prediction of the maximal Lyapunov exponent.

\section{INTRODUCTION}

Modern hadron colliders based on superconducting magnets suffer from the unavoidable effect of field-shape distortions, particularly harmful during the injection plateau. This critical period can last a large number of turns making difficult to evaluate the single-particle stability with computer simulations. In the case of the CERN Large Hadron Collider [1], the injection process will last for $10^{7}$ turns. On the other hand, numerical simulations based on symplectic tracking can hardly reach $10^{5}-10^{6}$ turns, also in consideration of the fact that a dense sampling of the phase space is crucial to obtain significant results. Three main approaches have been proposed in the past to speed-up the investigations on beam stability: the determination of the onset of chaotic behaviour using the maximal Lyapunov exponent [2,3], the evaluation of the drift in the space of approximated invariants carefully evaluated through numerical methods [4], and the visualization of the dynamic aperture reduction with increasing number of turns through survival plots $[5,6,7]$.

In this paper, we investigate the survival plots of the 4D Hènon map, with extended numerical methods.

The Hènon map [8] represents a simplified model of 4D betatronic motion where the coupling with longitudinal dynamics and the modulation of the linear frequencies are neglected. Using this map, we investigate the dynamics with numerical simulations and we propose phenomenological scenarios to interpret the results. We recall a way to define the dynamic aperture [9] and we compute it as a function of the number of turns $N$. The result can be interpolated with a three-parameter formula, justified in terms of the Nekhoroshev and KAM theorems. The interpolation fits very well with the numerical data and agrees with the prediction of the onset of chaos provided by the Lyapunov exponent. Our results can be extended to a more realistic accelerator model, describing 6D motion including the

* Work partially supported by EC Human Capital and Mobility contract Nr. ERBCHRXCT940480.

$\dagger$ Present address: CERN PS Division

\section{ANALYSIS OF THE PHASE SPACE}

We consider a grid of initial conditions on the plane $(x, y)$, with $p_{x}=p_{y}=0$ and we track the orbit for a large number of turns. From the tracking results, we evaluate the number of turns $N$ up to which the particle is stable and the two nonlinear frequencies $\left(\nu_{x}, \nu_{y}\right)$ of the motion. The frequencies are computed using an interpolation of the FFT plus Hanning filter [12]. For regular trajectories the frequencies are well defined with precision of the order of $1 / N^{4}$ [12]. For chaotic orbits instead the frequencies are not defined and the algorithm provides values that vary along the discrete time $N$, and that do not converge for $N \rightarrow \infty$.

The results are presented in various forms.

Long-term plot: each initial condition $(x, y)$ is plotted using a different marker according to the number of turns $N$ at which particle loss occurs.

Network of resonances: only the initial conditions $(x, y)$ locked on resonances are drawn: they satisfy

$$
q \nu_{1}+p \nu_{2}=l+\epsilon \quad q, p, l \in \mathbf{Z}
$$

We use the value $\epsilon=10^{-4}$. This plot directly displays the size of resonances, their position in phase space, and their relation with the dynamic aperture shown in the long-term plot.

Survival plot: the survival time $N$ is shown as a function of $D$, the average value of the initial conditions stable for $N$ turns.

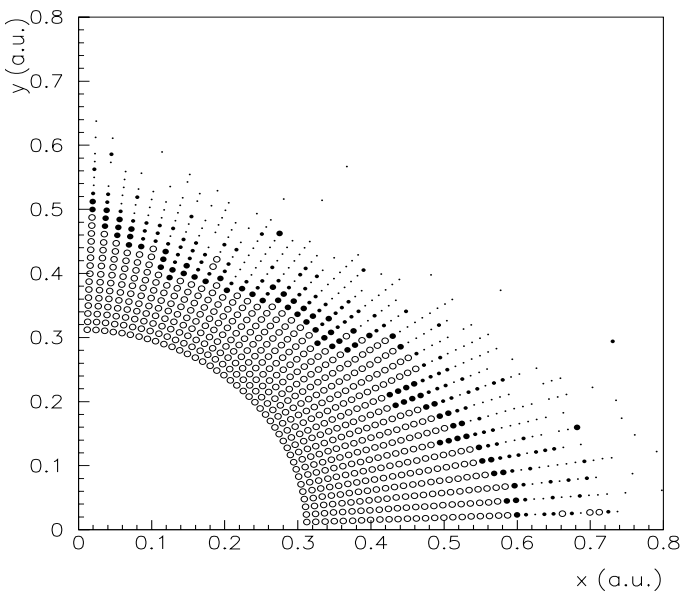

Figure 1: Long-term plot of the Hènon map at $\nu_{x}=0.168$, $\nu_{y}=0.201$ : empty circles represent initial conditions stable up to $10^{7}$ turns; full circles represent unstable initial conditions (smaller circles correspond to shorter stability times). 
In Fig. 1 a dense long-term plot for the Hénon map with linear frequencies $\nu_{x}=0.168, \nu_{y}=0.201$ is shown. A rectangular grid of initial conditions is iterated for at most $10^{7}$ turns. In the inner region all the particle are stable for at least $10^{7}$ turns. There are no holes, at least at the resolution used of our grid scan. After that, one finds a rather irregular but sharp border of instability: outside the border, one has a chaotic sea of initial conditions that are lost between $10^{6}$ and 10 turns.

In Fig. 2 the network of resonances for the same model of Fig. 1 is shown. One finds very large stable resonances; moreover, the mechanism of particle loss due to the diffusion along the resonant channels or due to resonance crossing does not seem to be very relevant. The bulk of longterm losses occurs in the wide chaotic band where no resonance structures are visible. This chaotic band is characterized by isolated points locked on low order resonances, that appear in the figure as a set of scattered dots. The same mechanism of loss has been observed for other values of the betatron frequencies and for other models, as a 4D model of the LHC [3].

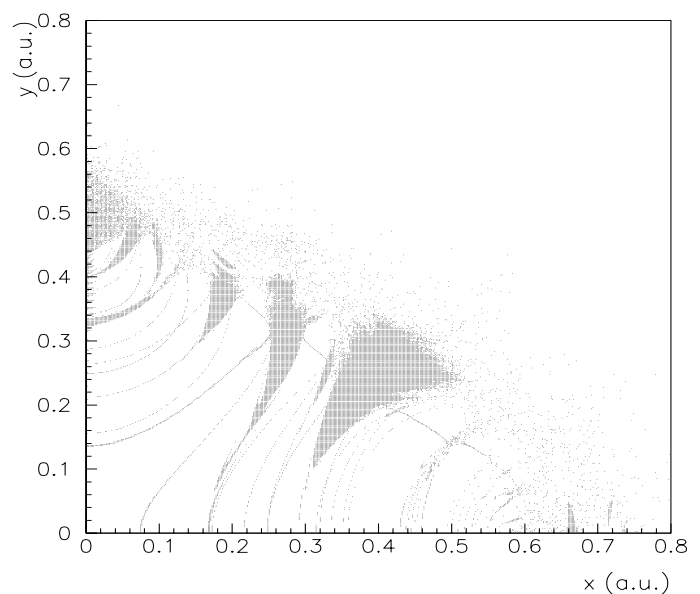

Figure 2: Network of resonances of the Hènon map at $\nu_{x}=$ $0.168, \nu_{y}=0.201$ : black dots represent initial conditions locked on resonances up to order 15 .

From these results we conclude that there is a rather sharp border that separates stable from unstable initial conditions for the considered number of turns $\left(10^{7}-10^{8}\right)$; long-term particle losses mainly occur in wide chaotic bands where all the integrable structure has been wiped out; the mechanism of diffusion along the resonant channels and due to resonance crossing are rather weak.

\section{DYNAMIC APERTURE AND ASSOCIATED ERRORS}

In a previous work [9] we defined the dynamic aperture as the average radius over the phase space area stable for $N$ turns. Particles are started along a 2D polar grid in the coordinate space $(x, y)$ :

$$
x=r \cos \theta \quad y=r \sin \theta
$$

with $p_{x} p_{y}$ equal to zero. Let $r(\theta ; N)$ be the last stable initial condition along $\theta$ before the first loss at a turn number lower than $N$ occurs. The dynamic aperture is

$$
D=\left(\int_{0}^{\pi / 2}[r(\theta ; N)]^{4} \sin 2 \theta d \theta\right)^{1 / 4}
$$

This definition differs from the usual one $[6,7]$, where, to speed up simulations, the scan is made only along the diagonal of the space $(x, y)$.

When the definition (3) is implemented in a computer code, one has to carry out two discretizations: one over the radial variable $r$ and one over the angular variable $\theta$. Let $\Delta r=\left(r_{\max }-r_{\min }\right) / N_{r}$ and $\Delta \theta=\pi / 2 N_{\theta}$ be the step size in $r$ and $\theta$ respectively. The total error associated with the dynamic aperture estimate can be obtained using gaussian sum in quadrature

$$
\Delta D=\sqrt{\left(\frac{\partial D}{\partial r} \frac{\Delta r}{2}\right)^{2}+\left(\frac{\partial D}{\partial \theta} \frac{\Delta \theta}{2}\right)^{2}} .
$$

To estimate the derivatives of $D$, we replace (3) with the simple average over $\theta$

$$
D=\frac{2}{\pi} \int_{0}^{\pi / 2} r(\theta ; N) d \theta \equiv<r(\theta ; N)>.
$$

Using this formula the associated error reads

$$
\Delta D=\sqrt{\frac{(\Delta r)^{2}}{4}+<\left|\frac{\partial r}{\partial \theta}\right|>^{2} \frac{(\Delta \theta)^{2}}{4}}
$$

Therefore, to optimize the integration steps, $\Delta r$ must be equal to $\Delta \theta$ times $\left\langle\left|\frac{\partial r}{\partial \theta}\right|>\right.$.

\section{PREDICTION BASED ON EXTRAPOLATION}

In Fig. 3 we show $D(N)$ versus $N$ for the same model of Fig. 1, carrying out simulations up to $10^{8}$ turns. A very fine phase space scan (120 radial steps from 0.3 to 0.8 and 60 angular steps) has been used in order to obtain a very high accuracy (error of the order of $1 \%$ ).

We interpolate the dynamic aperture with an inverse logarithmic law. Indeed, the phase space is divided into two regimes. An inner region where almost all the phase space is foliated into KAM tori, except a very small fraction where the Arnold diffusion can take place over the resonance web [14]. This region appears in simulations as a "full" domain of initial conditions stable for extremely high number of turns. Its average radius is called $D_{\infty}$. An outer region where almost all the foliation of phase space in KAM tori has been destroyed, and only a wide chaotic sea is left. Since we are close to the last KAM torus, we assume that in this region the particles escape to infinity with the rate provided by the Nekhoroshev estimate [15]:

$$
N(r)=N_{0} \exp \left(\frac{r_{*}}{r}\right)^{1 / \kappa},
$$

where $N(r)$ is the number of turns that are estimated to be stable for particles with initial amplitude smaller than $r$. 


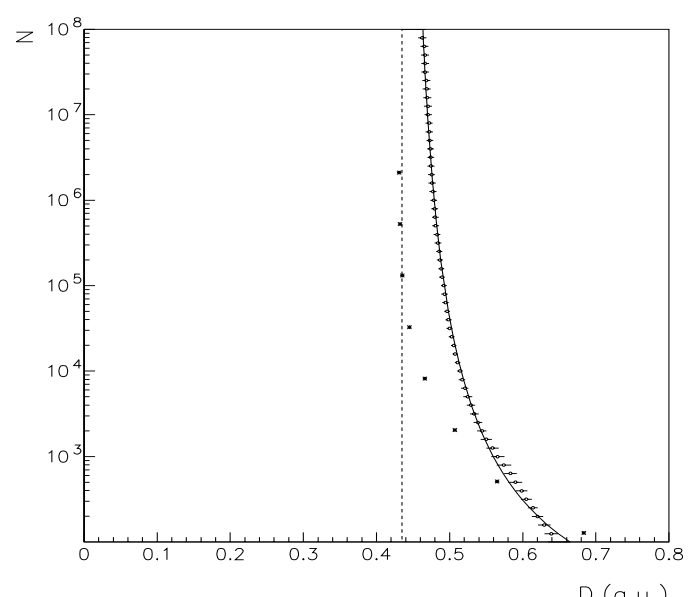

Figure 3: Dynamic aperture versus number of turns (dots) for the Hènon map at $\nu_{x}=0.168, \nu_{y}=0.201$; analytic interpolation (solid line) and extrapolation at infinity (dotted line). Prediction of the chaotic border according to the Lyapunov exponent (stars). The error bars are computed according to Eq. (6).

Using the information from KAM theorem (the existence of a positive $D_{\infty}$ ) and from the Nekhoroshev theorem (the inverse log decaying of the dynamic aperture), one obtains the following equation

$$
D(N)=D_{\infty}\left(1+\frac{b}{\log ^{\kappa}\left(N / N_{0}\right)}\right)
$$

We tried to interpolate the data shown in Fig. 3 with this formula using three free parameters $D_{\infty}, b$ and the exponent $\kappa$. We fixed $N_{0}$ to one by using the heuristic argument that $D(1)=\infty$. The solution is found by minimizing the value of the $\chi^{2}$ function, i.e.

$$
\chi^{2}=\frac{1}{J-3} \sum_{j=1}^{J} \frac{\left(D\left(N_{j}\right)-\hat{D}\left(N_{j}\right)\right)^{2}}{\sigma_{i}}
$$

where the interpolated dynamic aperture $\hat{D}\left(N_{i}\right)$ according to Eq. (8) is evaluated at the turn number $N_{i}$, and $\sqrt{\sigma_{i}}$ is the error estimated through Eq. (6). It turns out that it is rather difficult to determine the exponent with a high precision. For instance, if we consider all the exponents that provide a $\chi$ smaller than 0.7 , that corresponds in our case to a confidence level of $95 \%$, we obtain $\kappa \in[0.9,2]$. The optimal exponent for our case turns out to be around 1.5. The interpolation is shown in Fig. 3 as a solid line, and agrees very well with tracking data. Indeed, a refined version of the Nekhoroshev theorem [16] leads to the estimate $\kappa=(1+d) / 2$, where $d$ is the number of degrees of freedom of the particle motion. In our case we have $\kappa=1.5$. This is in agreement with our simulations, however the theoretical estimate of $\kappa$ is still quite controversial. For instance in the older Ref. [17, 18] the expected value of $\kappa$ for our case was estimated to be 3 . Additional checks for higher dimensions would be highly desirable in order to cross-check the optimal estimate of the exponent with the validity of our scenario.
We have also computed the estimate of the chaotic border through the Lyapunov exponent, using the same type of definition for the dynamic aperture, where now $r(\theta)$ is the amplitude of the particle immediately before the first particle along $\theta$ whose Lyapunov exponenet is greater than an appropriate threshold as described in Ref.[3]. The results are shown in Fig. 3. The Lyapunov guess of the chaotic border seems to converge rather rapidly (when compared to tracking) to the value of $D_{\infty}$.

Finally we evaluated the error on $D_{\infty}$ by computing the interval of $95 \%$ confidence level around the interpolating function (8), and we found $D_{\infty}=0.4750 \pm 0.0018$. The error is so small mostly because of the large number of turns used in the survival plot and of the fine grid scan applied in the evaluation of $D(N)$.

\section{ACKNOWLEDGEMENTS}

We wish to thank Prof. Turchetti for relevant contributions to the analysis and to the interpretation of the tracking data. Special thanks to S. Bongini, M. Böge, J. Ellison, J. Irwin, F. Schmidt and B. Warnock, for constructive discussions.

\section{REFERENCES}

[1] The LHC Study Group, CERN-LHC 95-05 (1995).

[2] Schmidt, F., Willeke, F., and Zimmermann, F., Part. Accel. 35249 (1991).

[3] Giovannozzi, M., Scandale, W., and Todesco, E., Part. Accel., 65-4 (1997) p.165.

[4] Warnock, R. L., and Ruth, R. D., Physica D 56188 (1992).

[5] Chao, A., AIP Conf. Proc. 230203 (1990).

[6] Yan, Y. SSC 500 (1991).

[7] Galluccio, F., and Schmidt, F., Third European Particle Accelerator Conference Gif sur Yvette: Edition Frontiéres (1993) pp. 640-42.

[8] Hénon, M., and Heiles, C., Astr. J. 69-1 73 (1964).

[9] Todesco, E., Giovannozzi M., Phys. Rev. E 534067 (1996).

[10] Böge, M., and Schmidt, F., these proceedings.

[11] Giovannozzi, M., Scandale, W., and Todesco, E., in preparation.

[12] Bartolini, R., Bazzani, A., Giovannozzi, M., Scandale, W., and Todesco, E., Part. Accel. 52147 (1996).

[13] Turchetti, G., private communication.

[14] Siegel, C. L., and Moser, J., Lectures in celestial mechanics, Berlin: Springer Verlag, 1971.

[15] Nekhoroshev, N., Russ. Math. Surv. 321 (1977).

[16] Bazzani, A., and Turchetti, G., Chaotic Dynamics: Theory and Practice New York: Plenum Press 59 (1991).

[17] Turchetti, G., Number theory and physics BerlinHeidelberg: Springer Verlag 223 (1990).

[18] Bazzani, A., Marmi S., and Turchetti, G., Cel. Mech. 47333 (1990). 